Vortex transport in a channel with periodic constrictions

This content has been downloaded from IOPscience. Please scroll down to see the full text. 2013 Supercond. Sci. Technol. 26095010

(http://iopscience.iop.org/0953-2048/26/9/095010)

View the table of contents for this issue, or go to the journal homepage for more

Download details:

IP Address: 146.175.11.111

This content was downloaded on 15/11/2013 at 13:37

Please note that terms and conditions apply. 


\title{
Vortex transport in a channel with periodic constrictions
}

\author{
A V Kapra ${ }^{1}$, D Y Vodolazov ${ }^{1,2}$ and V R Misko ${ }^{1}$ \\ ${ }^{1}$ Department of Physics, University of Antwerpen, Groenenborgerlaan 171, B-2020 Antwerpen, \\ Belgium \\ ${ }^{2}$ Institute for Physics of Microstructures, RAS, 603950 Nizhny Novgorod, Russia \\ E-mail: Vyacheslav.Misko@ua.ac.be
}

Received 9 May 2013, in final form 14 June 2013

Published 30 July 2013

Online at stacks.iop.org/SUST/26/095010

\begin{abstract}
By numerically solving the time-dependent Ginzburg-Landau equations in a type-II superconductor, characterized by a critical temperature $T_{\mathrm{c} 1}$, and the coherence length $\xi_{1}$, with a channel formed by overlapping rhombuses (diamond-like channel) made of another type-II superconductor, characterized, in general, by different $T_{c 2}$ and $\xi_{2}$, we investigate the dynamics of driven vortex matter for varying parameters of the channel: the width of the neck connecting the diamond cells, the cell geometry, and the ratio between the coherence lengths in the bank and the channel. We analyzed samples with periodic boundary conditions (which we call 'infinite' samples) and finite-size samples (with boundaries for vortex entry/exit), and we found that by tuning the channel parameters, one can manipulate the vortex dynamics, e.g., change the transition from flux-pinned to flux-flow regime and tune the slope of the $I V$-curves. In addition, we analyzed the effect of interstitial vortices on these characteristics. The critical current of this device was studied as a function of the applied magnetic field, $j_{\mathrm{c}}(H)$. The function $j_{\mathrm{c}}(H)$ reveals a striking commensurability peak, in agreement with recent experimental observations. The obtained results suggest that the diamond channel, which combines the properties of pinning arrays and flux-guiding channels, can be a promising candidate for potential use in devices controlling magnetic flux motion.
\end{abstract}

(Some figures may appear in colour only in the online journal)

\section{Introduction}

The critical current $j_{\mathrm{c}}(H)$ is the maximum current a superconductor can carry without dissipation. The first theoretical model to determine the critical current in a superconductor was the well-known Bean model [1]. The first 'microscopic' derivation of the Bean model, using a simple model of repelling vortices and pinning sites, was done by Richardson et al [2]. The dissipation is caused by vortex motion. Therefore, in order to increase the critical current, vortices should be either immobilized (i.e., pinned) or removed from the active area of a superconducting device. The former can be achieved by using various artificial pinning arrays including regular pinning arrays, e.g., square and triangular arrays of sub- $\mu \mathrm{m}$ holes (antidots) [3-14] or sub- $\mu \mathrm{m}$ $\mathrm{Ni}$ triangles on top of $\mathrm{Si}$ substrate [15] or blind antidots (i.e., holes which partially perforate the film to a certain depth) [16] and pinning arrays with field-dependent pinning strength [17]. Even a few individual pinning sites arranged at proper positions can be very efficient in trapping undesired vortices. For example, it was demonstrated [18] that the low-frequency noise due to vortex motion in high-temperature superconducting quantum interference devices (SQUIDs) can be strongly reduced by a 'strategic' arrangement of antidots patterned into the SQUID. Alternatively, it was recently proposed to use aperiodic pinning arrays instead of periodic arrays. Despite the fact that they are incommensurate with the (undistorted) vortex lattice, these pinning arrays were proven to be even more efficient in magnetic flux pinning than regular arrays. The enhancement of the pinning strength in a broad range of magnetic fields is provided by elastic deformation of a vortex lattice, as was recently demonstrated theoretically $[19,20]$ and experimentally [21-23, 26] for quasiperiodic Penrose-tiling pinning arrays and for graded pinning arrays [24, 25]. 
Another efficient method of the critical current control in superconducting nano- and micro-devices is to remove magnetic flux from the active area of the device. For this purpose, it was proposed to use various realizations of vortex ratchets, e.g., fluxon pumps and lenses [27] using a 'funnel' channel [28], voltage rectifiers [29], and Josephson vortex ratchets [30]. Thus it was shown that vortex ratchets can be used for vortex removal (or significant reduction of number) from a superconductor [15, 31-34]. Also, the effect of the field history on the vortex dynamics in narrow ratchet channels was investigated [35]. It was shown that the edge barrier controls the critical current in a thin film of a weak-pinning superconductor [36] and in a similar system with periodic constrictions [37].

In addition, the motion of the magnetic flux can be controlled using the vortex guidance effect [7, 38-40], which manifests itself in the flux motion along the symmetry directions of an array of pinning sites. It was shown [38] that the vortex lattice undergoes a series of locking transitions when increasing the applied driving force. As the vortex lattice passes through these phases, the transverse velocity component as a function of increasing transverse drive shows a series of plateaus which form a devil's staircase structure. Experimentally, controlled trapping and guided motion of vortices via special arrangements of antidots in $\mathrm{YBa}_{2} \mathrm{Cu}_{3} \mathrm{O}_{7}$ films was demonstrated using resistive Hall-type measurements. In contrast to conventional vortex motion due to the vortex unpinning at currents exceeding the critical current, this motion was present down to zero current and low temperatures and it was characterized by a linear voltage-current dependence, i.e., Ohmic behavior [7]. Magneto-optical imaging measurements of a $\mathrm{YBa}_{2} \mathrm{Cu}_{3} \mathrm{O}_{x}$ film shaped as a long strip with perpendicular antidot arrays revealed [40] strong guidance of flux and large perturbations of the overall flux penetration and flow of current.

When driven across the guiding channel, vortices display a very unusual dynamics. Thus in [41] the motion of vortices confined to a straight pin-free channel in a strong-pinning superconducting sample was studied. It was found that, when a constant current is applied across this system, a very unusual oscillatory shearing appears, in which the vortices moving at the edges of the channel periodically trail behind and then suddenly leapfrog past the vortices moving in the inner rows.

Recently, the effect of magnetic flux guidance in a hybrid superconductor magnetic-dot-array bilayer was theoretically analyzed, using Langevin-type molecular-dynamics simulations [42] and the time-dependent Ginzburg-Landau equations [43]. In particular, the vortex-antivortex dynamics was analyzed for different arrangements and magnetic moments of the dipoles, as a function of the angle between the direction of the magnetic dipole and that of the Lorentz force produced by the applied current. It was shown that the interplay of the attractive interaction between a vortex-antivortex pair and the Lorentz force resulted either in separation of (anti)vortices and their motion in opposite directions or in their annihilation. The guided motion effect was analyzed [43] in finite and in infinite samples. It was shown that in finite samples the magneticdipole-induced vortex-antivortex guidance is influenced by the self-interaction of the vortex-antivortex pairs with their images, while in a periodic array of dipoles the guidance is determined by the interaction of a vortex-antivortex pair with other dipoles and vortex-antivortex pairs created by them.

In this work, we investigate the vortex dynamics in a system consisting of a superconductor with a channel made of another superconductor. The channel is formed by compartments in the shape of rhombuses ('diamonds') partially overlapped such that they form a continuous channel (in the limiting case, adjacent 'diamonds' touch each other just at one point). Our choice of the channel geometry was motivated by the recent experiment [37] on vortex dynamics in channels with periodic constrictions. The authors measured the critical current $J_{\mathrm{c}}$ in diamond-cell channels and found reversible oscillations in $J_{\mathrm{c}}$ as a function of the applied magnetic field [37]. The goal of our study is the understanding of: (i) the dynamics of moving vortices in detail and (ii) the possibility of manipulation of this dynamics by tuning the geometric and material parameters of the channel (which is hardly accessible in experiments). We analyze the vortex dynamics in a diamond-cell channel from the point of view of pinning properties of the system and the possibility of manipulating the dynamical regimes (e.g., flux-pinned regime and flux-flow regime). As we show below, each individual compartment of the channel acts as a 'soft-pinning' site which is the key property of this system. The 'magnetic core' of a vortex (i.e., the region with the radius equal to the magnetic field penetration depth $\lambda$, where the screening current of the vortex decreases from the maximum value to approximately zero) extends outside the channel, and by changing the degree of overlap of the diamond cells (and thus the minimum width of the channel at the neck between neighboring compartments), one can manipulate the pinning strength. As we further show in this work, this provides a flexible tool for controlling the dynamical regimes in this system. On the other hand, the diamond channel acts as a guiding 'row of pinning sites' but appears to be more efficient than an array of separate pinning sites, due to the partial overlap of the pinning potentials created by each diamond cell.

To study the vortex dynamics, in this work we rely upon the time-dependent Ginzburg-Landau equations (TDGL) which take into account the influence of the cell confinement on the vortex motion. We investigate the equilibrium and transport properties of driven vortices. In particular, we study the flux motion in the diamond channel for different material parameters and geometries of the channel and calculate appropriate $I V$-curves and the critical current, $j_{\mathrm{c}}(H)$, as a function of the applied magnetic field. We found that the critical current, $j_{\mathrm{c}}(H)$, demonstrates striking commensurability effect, in agreement with the experimental observations [37]. Analyzing the $I V$-curves, we show that, by varying the material parameters (i.e., the ratio of the coherence lengths inside and outside the channel, $\xi_{2} / \xi_{1}$ ), the 'gap' between the diamond cells (i.e., the minimum channel width, $w$ ) and the geometry of the cell, one can manipulate the dynamical regimes in the diamond channel, e.g., switch between the flux-pinning and flux-flow regimes, and control the slope of the corresponding $I V$-curves. 
The pinning properties of the system are related to the magnetic core pinning. Despite the fact that the vortex core can be very small (e.g., $\xi \sim 1-5 \mathrm{~nm}$ in HTSC), its supercurrent spreads over a wide range $(\sim \lambda \gg \xi)$. Therefore, if we construct a magnetic barrier with an opening $w$, such that $\lambda>w>\xi$ than the vortex interaction with the opening edges would lead to pinning. Also the opening $w$ influences the surface barrier energy, which leads to a vortex generation for lower values of magnetic field (in finite-size samples). The diamond channel confines the vortex motion in the transverse $(y-)$ direction. On the other hand, the modulation of the channel width introduces pinning in the $x$-direction. Our calculations show that by adjusting the ratio $\xi_{2} / \xi_{1}$ and the external magnetic field, one can maintain the necessary amount of vortices in the channel. This provides opportunities for studying the interaction between satellite (bank) vortices and vortices in the channel.

The paper is organized as follows. In section 2 we introduce the model system. In section 3 , we describe the mechanism of vortex generation in the channel for two different system preparation regimes, i.e., field-cooled and zero-field-cooled regime, and we analyze equilibrium vortex states in finite and 'infinite' (i.e., with periodic boundary conditions) samples. In section 4, we discuss the flux dynamics in the diamond channel, including the analysis of the $I V$-curves for varying material parameters and the channel geometry, the effect of commensurability observed in the critical current $j_{\mathrm{c}}(H)$. The conclusions are presented in section 5 .

\section{Model}

Our system consists of a type-II SC film characterized by the coherence length $\xi_{1}$ with a channel formed by diamond-shape cells of another type-II SC with a larger coherence length $\xi_{2}: \xi_{2}>\xi_{1}$. Our model channel is shown in figure 1 . This configuration provides a vortex-flow channel with modulated width, the so-called diamond-cell channel [37]. The ratio between the coherence lengths in the channel and in the bank superconductor, $\xi_{2} / \xi_{1}$, is a variable parameter, typically taken as $\xi_{2} / \xi_{1}=6$. The bottleneck $w$ (see figure 1) of the channel varies from 0 to $8 \xi_{1}$. The ratio $\xi_{2} / \xi_{1}$ mainly controls the vortex mobility in the channel. By changing the width of the channel and the ratio $\xi_{2} / \xi_{1}$, we can manipulate the vortex motion inside it. An external homogeneous magnetic field generates vortices in the channel which are driven by an external transport current.

We consider samples either finite or infinite in the $x$-direction. To model an infinite channel, we use periodic boundary conditions applied to the phase and the amplitude of the SC order parameter. In our simulations, we typically consider samples with dimensions $80 \xi_{1} \times 50 \xi_{1}$ and $20 \xi_{1} \times$ $50 \xi_{1}$, where $\xi_{1}$ is the superconducting coherence length at zero temperature $T$. We use the field-cooled regime (FC) and then (after some relaxation time) we turn the transport current on and investigate the vortex motion.

In order to avoid the influence of the metal leads on channel vortices and study the interaction of vortices in the

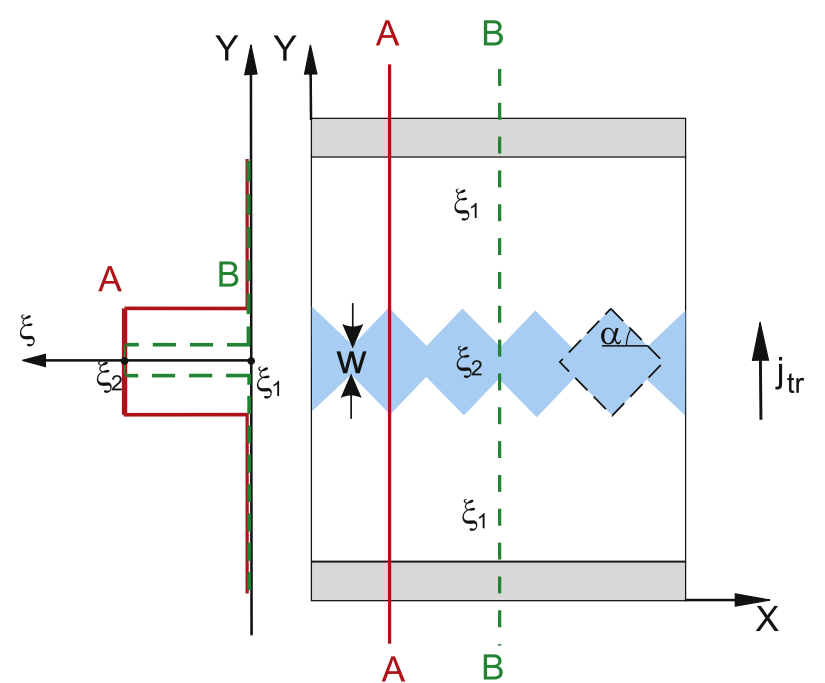

Figure 1. Sketch of the model system. The 'diamond channel' is filled by blue (gray). The channel is characterized by the coherence length $\xi_{2}$, while the main superconductor (bank) has $\xi_{1}$. The channel parameters (shown in the sketch) are: the ratio $\xi_{2} / \xi_{1}$, the minimum width of the channel, $w$, and the cell shape characterized by angle $\alpha$.

channel with satellite vortices (i.e., out-of-channel), we set the boundaries of the film $\sim 30 \xi_{1}$ away from the each side of the diamond channel (in the $y$-direction). Note that for $\xi_{2} / \xi_{1}=1$ we observe the behavior typical for a homogeneous SC film.

After the initial vortex configuration in the system is prepared, the current is turned on, and we investigate the vortex-antivortex ( $\mathrm{v}-\mathrm{av})$ dynamics. To study the dynamics of the v-av pairs, we employ the time-dependent Ginzburg-Landau equation [44, 45]:

$$
\begin{gathered}
u\left(\frac{\partial}{\partial t}+\mathrm{i} \varphi\right) \psi=\xi_{i}(\nabla-\mathrm{iA})^{2} \psi+\left(1-|\psi|^{2}\right) \psi, \\
\xi_{i}= \begin{cases}\xi_{1}, & \text { in the bank SCs } \\
\xi_{2}, & \text { in the channel. }\end{cases}
\end{gathered}
$$

The default values are: $\xi_{2}=6$ inside the diamond channel and $\xi_{1}=1$ in the bank. The equation is to be solved self-consistently with the Poisson equation for the electrostatic potential,

$$
\Delta \varphi=\operatorname{div}\left(\operatorname{Im}\left(\psi^{*}(\nabla-\mathrm{iA}) \psi\right)\right) .
$$

In equations (1) and (2), all the physical quantities are expressed in dimensionless units: temperature $T$ in units of the critical temperature $T_{\mathrm{c}}$, the vector potential $\mathbf{A}$ in units $\Phi_{0} /(2 \pi \xi(T))$ (where $\Phi_{0}$ is the quantum of magnetic flux), the order parameter in units of $\Delta_{0}=4 k_{\mathrm{B}} T_{\mathrm{c}} u^{1 / 2} / \pi(1-$ $\left.T / T_{\mathrm{c}}\right)^{1 / 2}$, and the length in units of the coherence length $\xi(T)=\left(8 k_{\mathrm{B}} T_{\mathrm{c}} / \pi \hbar D\right)^{-1 / 2} /\left(1-T / T_{\mathrm{c}}\right)^{1 / 2}$. Using these units, the magnetic field is scaled by $H_{\mathrm{c} 2}=\Phi_{0} / 2 \pi \xi(T)^{2}$ and the current density by $j_{0}=\sigma_{\mathrm{n}} \hbar / 2 e \tau_{\mathrm{GL}}(T) \xi(T)$. Time is scaled in units of the Ginzburg-Landau relaxation time $\tau_{\mathrm{GL}}(T)=$ $\pi \hbar / 8 k_{\mathrm{B}} T_{\mathrm{c}} u /\left(1-T / T_{\mathrm{c}}\right)$, the electrostatic potential $\varphi$ in units of $\varphi_{0}=\hbar / 2 e \tau_{\mathrm{GL}}(T)$, where $\sigma_{\mathrm{n}}$ is the normal-state conductivity, and $D$ is the diffusion constant. Parameter $u$ governs the time change of $|\psi|$ and the length of penetration of the electric field 
into a superconductor [46]. Since we are interested mainly in the dynamics of (anti-)vortex motion but not in the time evolution of the $\mathrm{v}-\mathrm{av}$ nucleation and annihilation, for our problem the actual value of $u$ does not play an essential role, and we choose $u=5.79$ (note that this value depends on the specific superconductor).

We assume that the thickness of the superconducting film $d_{\mathrm{s}}$ is smaller than the effective magnetic field penetration depth, $\Lambda=\lambda(T)^{2} / d_{\mathrm{s}}$, where $d_{\mathrm{s}}$ is the thickness of the sample, and therefore we can neglect the influence of the screening and transport currents on $\mathbf{A}$ [47].

For convenience, we shift the diamond channel to the left for a half of a diamond-cell length in the $x$-direction. In this case, the first and the last cells are half-cells. This decreases the energy for vortex entry into the film and exit out in case of finite samples. It also stabilizes the vortex motion inside the channel (jamming in the last cell does not appear and does not force vortices to leave the channel). In the case of infinite sample this cell shift does not play an essential role due to the periodic boundary condition.

In the $x$-direction, we use periodic boundary conditions for the magnitude and phase of the order parameter: $\left.\psi\right|_{0}=$ $\left.\psi\right|_{\mathrm{N}},\left.\varphi\right|_{0}=\left.\varphi\right|_{\mathrm{N}}$, and in the $y$-direction we employ the 'normal metal-superconductor' boundary conditions: $\psi=$ $0, \partial \varphi / \partial n=-j_{\mathrm{n}}$. The current is applied along the $y$-axis as shown in figure 1 . We define the critical 'depinning' current $j_{\mathrm{c}}$ as the current resulting in non-zero vortex velocity in the dynamic regime. The typical value of the critical voltage corresponds to $1.0 \times 10^{-4} \varphi_{0}$.

Numerical solution of the TDGL equations is obtained using the finite-difference method, Fourier analysis and the cyclic reduction method (FACR) [48-50].

\section{Equilibrium vortex states}

In this section we describe how the initial state of the system is prepared. In our simulations, we mimic two experimental regimes for initial state preparation: field-cooled (FC) regime and zero-field-cooled (ZFC) regime [51]. In the former case, we start the simulation from the normal state and let the system relax in the presence of a homogeneous magnetic field. The applied magnetic field generates vortices in the sample. In the latter case, the system is cooled down without field, and then the field penetrates the system through its boundary in the form of vortices.

\subsection{Finite samples}

Here we analyze equilibrium vortex states in finite samples obtained for various values of the external magnetic field. These states were obtained using the ZFC regime. After the cooling, vortices enter the sample through its boundaries. The mechanism of the current-assisted vortex entry is as follows. The total current (i.e., the geometric sum of the screening and the transport currents) near one of the boundaries becomes higher than the depairing current (see figure 2). This lowers the surface barrier for vortex entry. An additional factor for the barrier decrease is the fact that the coherence length in

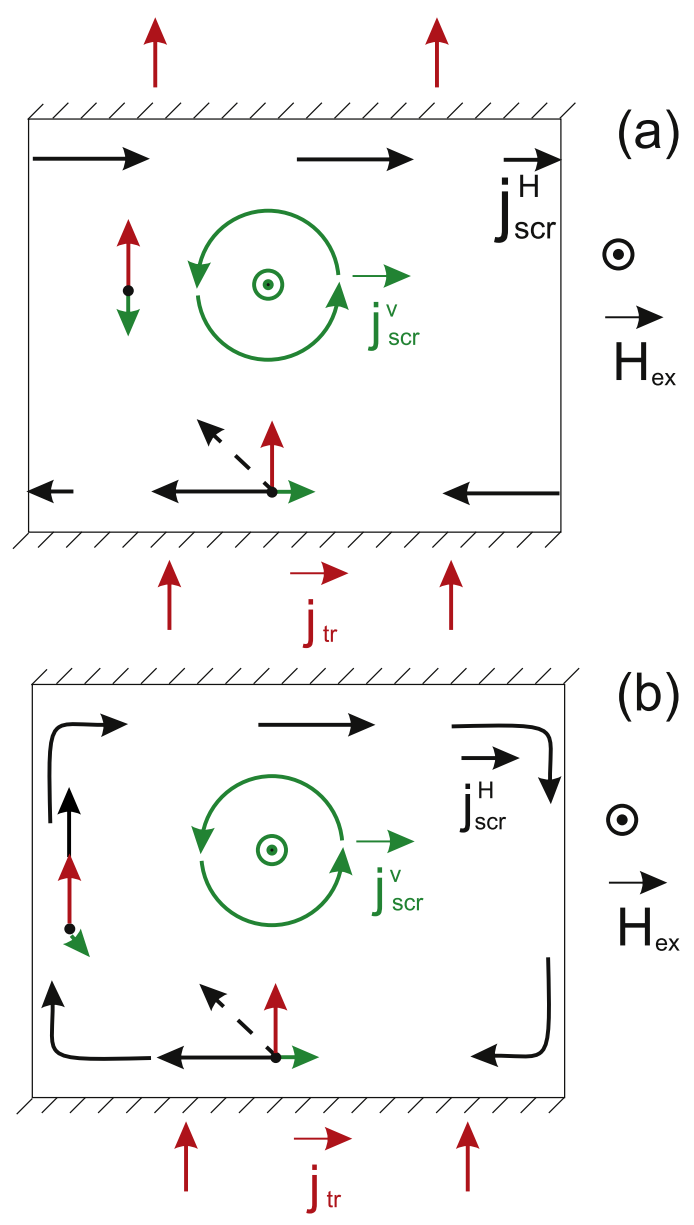

Figure 2. Current distribution in an infinite (a) and finite (b) superconducting sample with a vortex inside. Solid black arrows represent the surface screening current, $j_{\text {scr }}^{H}$; solid red (dark gray) the transport current, $j_{\mathrm{c}}$; solid green (light gray) the current of the vortex, $j_{\mathrm{scr}}^{\mathrm{v}} . H$ shows the direction of the external magnetic field (out-of-plane). The dashed-line vector shows the total current of the above three contributions at a point near the boundary (marked with a black dot).

the channel $\xi_{2}$ is larger than that outside $\xi_{1}$. These two factors provide a condition for vortex entry. Each new vortex which enters the channel increases the barrier since its current lowers the total sum of the transport and the screening currents near the film edge (see figure 2(b)). Vortices will enter and occupy the channel until the vortex currents rise the barrier high enough, resulting in a balance between the flux outside and inside the sample (figure 3(a)). Due to the condition $\xi_{2} / \xi_{1}>$ 1 , vortices will preferably occupy the channel rather than the bank. Therefore, the diamond channel serves as a pinning channel.

The excess of the flux which is not trapped by the channel enters the bank (i.e., the superconductor with a lower coherence length $\left.\xi_{1}<\xi_{2}\right)$ in the form of additional (i.e., not 'pinned') vortices. Figures 3(b) and (c) represent equilibrium vortex states in the diamond channel for a larger external magnetic field than in case (a). The figures show that the vortices inside the channel impose the symmetry on the interstitial vortices (the situation is opposite to the one described in [37], where the interstitial vortices imposed 

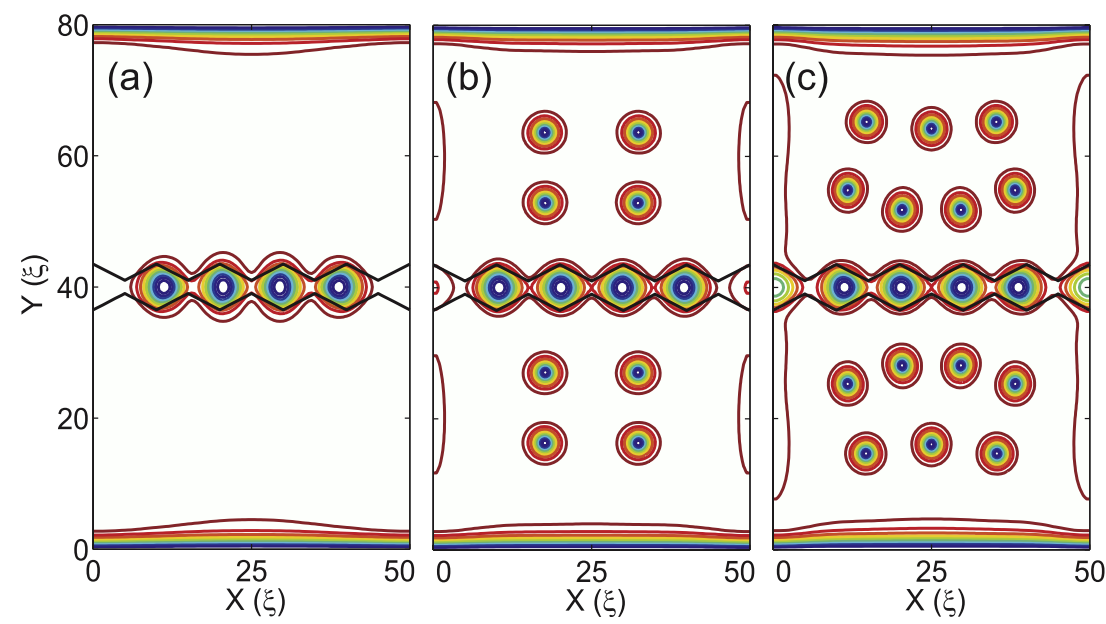

Figure 3. Initial (static) vortex configurations (i.e., in the absence of driving) in the sample $\left(w=4 \xi_{1}\right.$ and $\left.\xi_{2} / \xi_{1}=6\right)$ for the external magnetic field: (a) $H=0.022 H_{\mathrm{c} 2}$; (b) $H=0.04 H_{\mathrm{c} 2}$; (c) $H=0.055 H_{\mathrm{c} 2}$.

the symmetry on the channel vortices). This is a direct consequence of the absence of the intrinsic pinning. At large magnetic field $H=0.055 H_{\mathrm{c} 2}$ (figure 3(c)), the interstitial vortices are tightly packed and the interaction between them overcomes the interaction with the channel vortices. This leads to the appearance of an Abrikosov-type lattice of interstitial vortices.

In mesoscopic SCs close to the normal state, the boundary conditions induce the order parameter to follow the symmetry imposed by the sample geometry as demonstrated, e.g., for mesoscopic disks [52-55], triangles [56, 57], and stars [58]. It explains the absence of triangular vortex lattice outside of the diamond channel.

In figure 4 , we show equilibrium vortex states for varying $\xi_{2} / \xi_{1}$. The ratio $\xi_{2} / \xi_{1}$ governs the surface barrier for vortex entry into the diamond channel. The larger the ratio, the lower the barrier and the stronger the pinning inside each cell. For the same external magnetic field, a smaller $\xi_{2} / \xi_{1}$ value (surface barrier) leads to a smaller total vortex number in the film, and a weaker pinning leads to a smaller vortex number in the channel itself.

These results show that, by varying $\xi_{2} / \xi_{1}$, one can manipulate the ratio of the vortex number in the channel and outside the channel. This manipulation is possible for relatively high magnetic fields $\left(H \gtrsim 0.03 H_{\mathrm{c} 2}\right)$ when the channel cannot trap all the vortices, and they will penetrate outside of the channel and will vary the ratio between the channel and the interstitial vortices. For low magnetic fields $\left(H \lesssim 0.03 H_{\mathrm{c} 2}\right)$, vortices occupy the channel until it traps them inside, and no interstitial vortex appears. Analyzing the vortex dynamics in the diamond channel, we are interested in regimes when vortices are mainly trapped in the channel.

\subsection{Infinite sample}

Infinite samples are modeled by employing periodic boundary conditions in the $x$-direction of the simulation cell. Initial vortex configurations are prepared by simulating the FC regime. Equilibrium vortex states in the diamond channel for
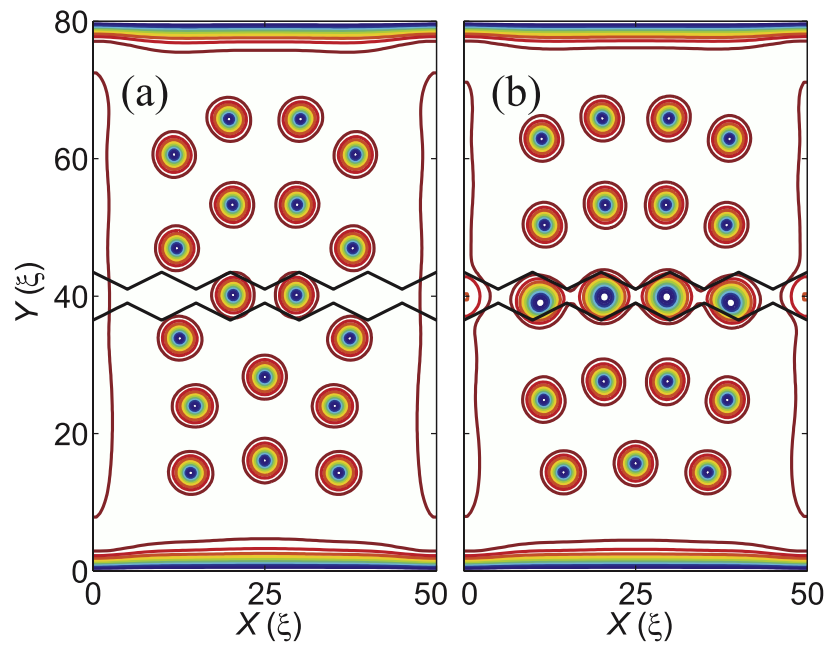

Figure 4. Initial (static) vortex configurations (i.e., in the absence of driving) in the sample ( $w=4 \xi_{1}$ and $\left.H=0.055 H_{\mathrm{c} 2}\right)$ for the ratio of the coherence lengths: (a) $\xi_{2} / \xi_{1}=1$; (b) $\xi_{2} / \xi_{1}=3$.

various values of the external magnetic field are presented in figure 5. The corresponding current distribution is shown in figure 2(a). Note that the periodic boundary condition results in a more homogeneous vortex distribution in the $x$-direction than in finite-size samples.

In our simulations the vortex number in a stationary state does not depend on the ratio $\xi_{2} / \xi_{1}$ (as examined for $H=$ $\left.0.01-0.1 H_{\mathrm{c} 2}\right)$. Despite this, $\xi_{2} / \xi_{1}$ becomes significant in the dynamical regime as it is included in the kinetic term of the TDGL equations (1). Therefore, a variation in $\xi_{2} / \xi_{1}$ governs the ratio between the vortex number in the channel and out of the channel in the dynamic regime.

\section{Flux dynamics}

In this section, we study the vortex dynamics driven by external current. As we demonstrated in section 3, vortices can occupy either the channel area only (see figure 3(a)) or 


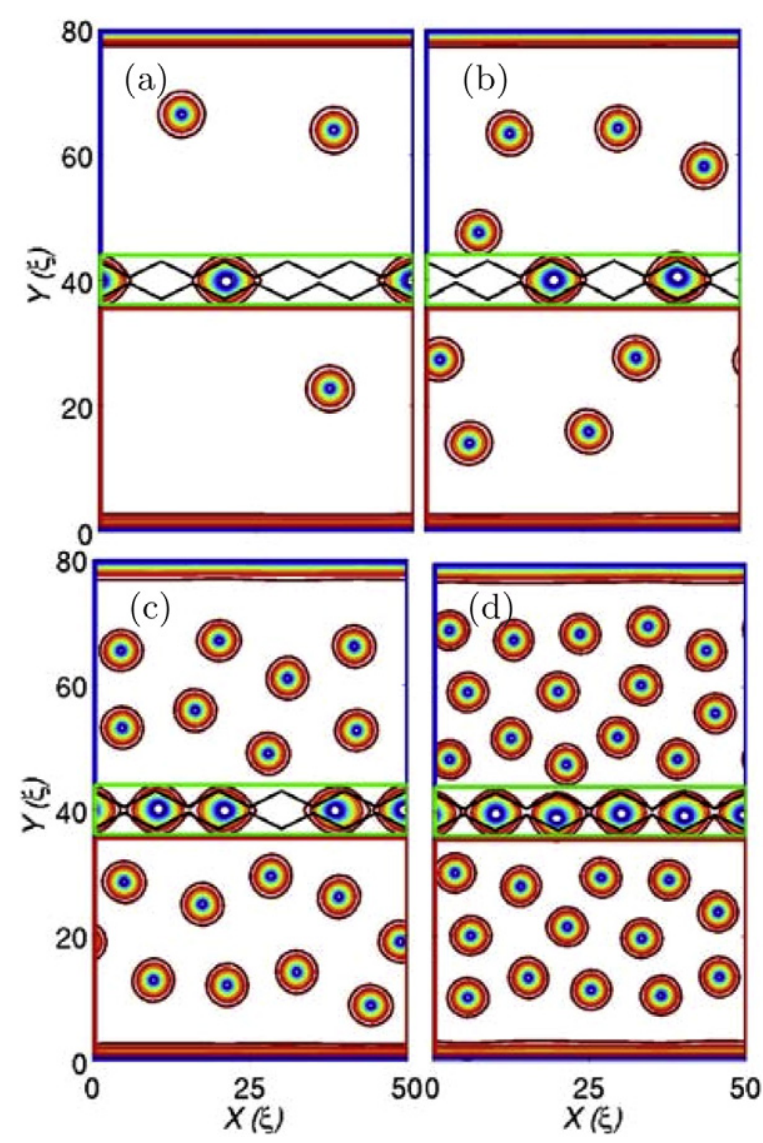

Figure 5. Initial (static) vortex configurations (i.e., in the absence of driving) in the sample ( $w=2 \xi_{1}$ and $\left.\xi_{2} / \xi_{1}=6\right)$ for the external magnetic field: (a) $H=0.01 H_{\mathrm{c} 2}$; (b) $H=0.02 H_{\mathrm{c} 2}$; (c) $H=0.04 H_{\mathrm{c} 2}$; (d) $H=0.06 H_{\mathrm{c} 2}$.

the channel and the bank area (figures 3(b) and (c)), depending on the applied magnetic field. Correspondingly, the dynamical behavior will differ for these two different cases.

For low magnetic fields, which correspond to the case when vortices occupy only the diamond channel, the motion of vortices occur in the form of a 'single file', i.e., along a single straight-line trajectory inside the channel, following one another, without permutations. This motion can be controlled, as we show below, by the geometry of the channel.

For higher magnetic fields, which provide generation of vortices both in the channel and in the banks, the dynamical behavior is different. The contribution to the total vortex current will be provided by vortices moving inside the channel and interstitial vortices. Due to the difference in the material parameters, i.e., $\xi_{1}$ and $\xi_{2}$, the vortices in the channel move faster then interstitial vortices. However, the velocity of the vortices in the channel can be manipulated by changing the channel geometry which results in a variety of dynamical regimes. Examples of snapshots of moving vortices in the system, for magnetic field $H=0.02 H_{\mathrm{c} 2}$ and $\xi_{2} / \xi_{1}=6$, are shown in figure 6 . It is interesting to note that, while in the initial state (without current) the vortex arrangement can be less ordered (figure 6(a)), the system of vortices becomes more ordered during the motion (figure 6(b)). This occurs due to the fact that channel vortices move faster than interstitial
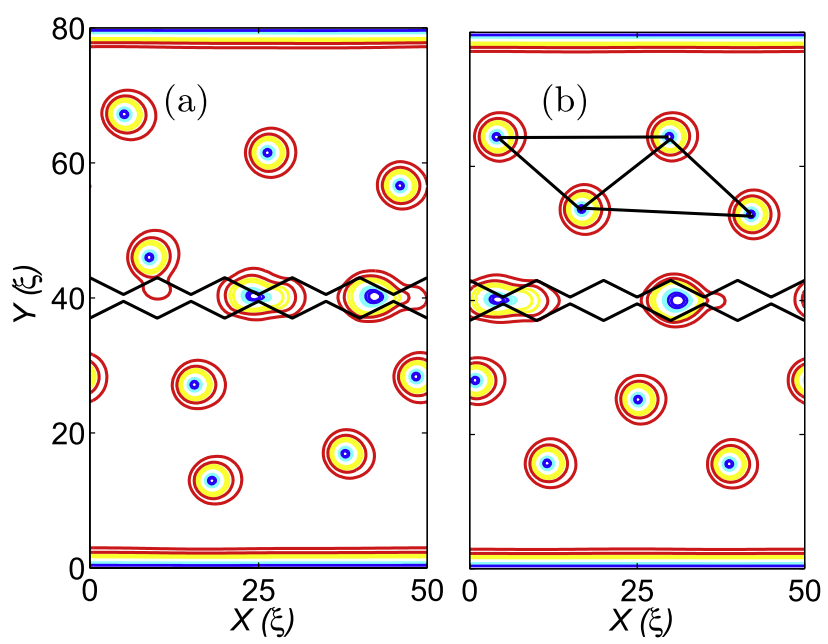

Figure 6. Snapshots of driven vortex matter in a diamond channel in an infinite sample: $\left(\xi_{2} / \xi_{1}=6, w=2 \xi_{1}\right)$ at times: (a) $t=235 \tau_{\mathrm{GL}}$ (b) $t=1515 \tau_{\mathrm{GL}}$. The external magnetic field is $H=0.02 H_{\mathrm{c} 2}$. The solid black lines, forming triangles, show the dynamically ordered 'lattice' of moving interstitial vortices.

vortices, and after some time the system adjusts itself to minimize its energy such that the interstitial vortices becomes more ordered (i.e., the dynamical ordering).

\subsection{The IV-curves of the system}

The above difference in the dynamical behavior of the system without (i.e., vortices are only in the channel) and with interstitial vortices is illustrated by the $I V$-curves. In figure 7 , we plot $I V$-curves for the channel vortices (figure 7(a); for convenience, we consider a narrow channel, i.e., with width $d_{y}=20 \xi$, in order to avoid the appearance of interstitial vortices) and for channel and interstitial vortices (figure 7(b)).

To reveal the effect of the channel geometry on the vortex dynamics, we calculate $I V$-curves for varying channel gap $w$ (see figure 1). In the case of channel vortices, they are all confined to the channel, and a small gap results in vortex pinning by the diamond cells. The limiting case $w=$ 0 corresponds, obviously, to the maximum vortex pinning. As a result, this situation is characterized by the maximum critical current and extended region of flux-pinning regime (figure 7(a)). The critical value corresponds to the 'depinning' current, when a vortex overcomes the potential barrier and jumps from one diamond cell to the adjacent cell.

Opening the gap allows an easier vortex transfer between the cells which in turn results in a lower critical current (compare the $I V$-curves in figure 7(a) for $w=4 \xi$ and $w=8 \xi$ ). It is worth noting, however, that even for $w>\xi$ vortices remain pinned by the diamond cells, due to magnetic core pinning [36]. Indeed, the magnetic core of a vortex extends outside the channel, and the variation of the channel width determines the profile of the vortex supercurrent which is associated with a change in the energy of the vortex. This 'soft-pinning' regime considerably enhances the tunability of the diamond-channel 'pinning array', in comparison with usual pinning arrays (i.e., consisting of separate pinning sites). 

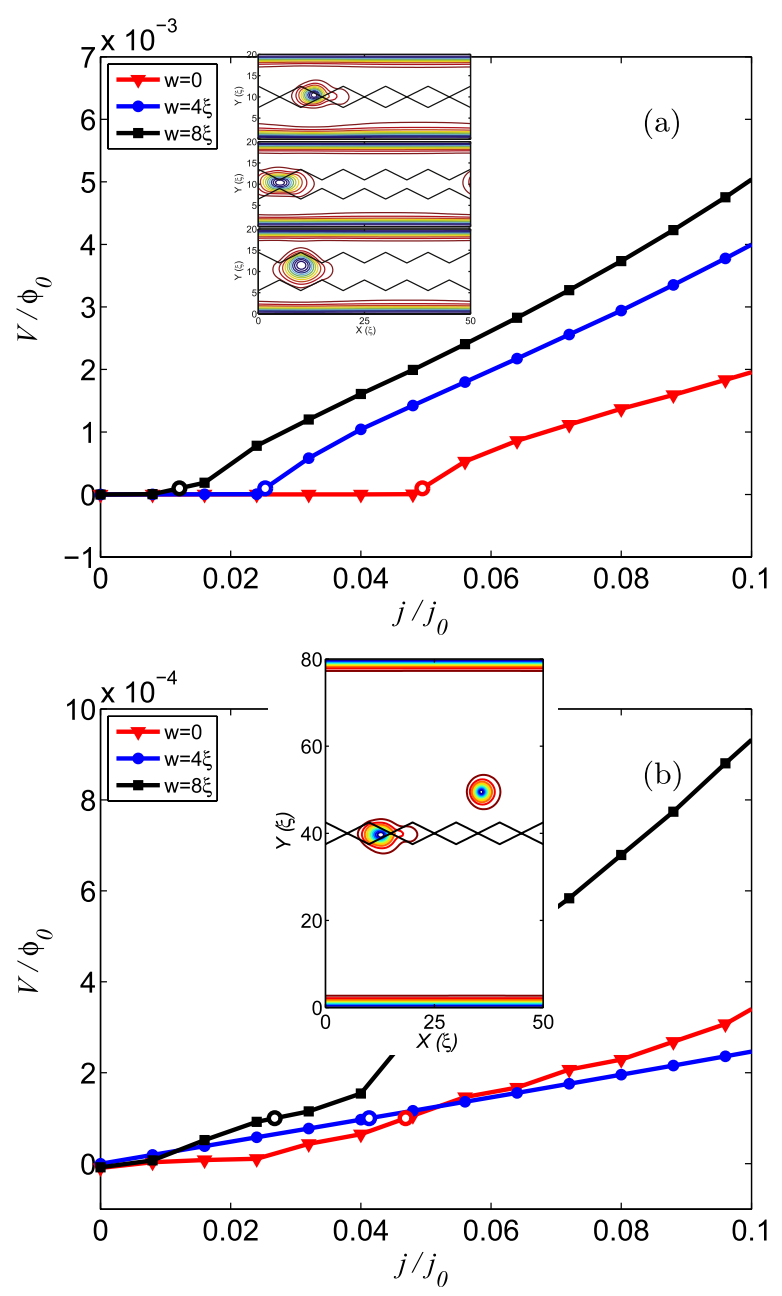

Figure 7. The $I V$-curves of the system $\left(\xi_{2} / \xi_{1}=6, \operatorname{tg}(\alpha)=0.5\right)$ for different sizes of the simulation cell (in an infinite sample):

(a) $50 \xi \times 20 \xi$ with one vortex in the channel, which corresponds to external magnetic field $H=0.035 H_{\mathrm{c} 2}$ (for $w=0$ ), $H=0.025 H_{\mathrm{c} 2}$ (for $w=4 \xi$ ), $H=0.025 H_{\mathrm{c} 2}$ (for $w=8 \xi$ ). The critical current is: $j_{\mathrm{c}}=0.012 j_{0}, j_{\mathrm{c}}=0.025 j_{0}$, and $j_{\mathrm{c}}=0.049 j_{0}$, correspondingly. The insets show the distribution of the square modulus of the order parameter in the sample for the above channel configurations, $w=0,4$, and $8 \xi$. (b) $w=50 \xi \times 80 \xi$ with one vortex in the channel and one interstitial vortex. The inset shows the order parameter in the cell with channel gap $w=0$ at driving current $j=0.048 j_{0}$, and magnetic field $H=0.004 H_{\mathrm{c} 2}$. (A contour plot for $w=4 \xi$ and $w=4 \xi$ is similar.) The critical current $j_{\mathrm{c}}$ (estimated using the voltage threshold criterion, $V_{\mathrm{c}} / \phi=10^{-4}$ ) is $0.027 j_{0}, 0.041 j_{0}$, and $0.047 j_{0}$, correspondingly.

As a result, by changing the gap, $w$, we can gradually change the critical current of this system and thus manipulate the transition from the flux-pinned to the flux-flow regime. As shown above, for high enough fields vortices can enter the banks (i.e., the area outside the diamond channel). Therefore, it is interesting to analyze the effect of interstitial vortices on the vortex dynamics in our system.

As expected, the presence of additional vortices will strongly suppress the flux-pinning regime, since interstitial vortices can flow freely for any low applied current. A non-zero critical current may appear only as a result of caging of interstitial vortices due to their interaction with

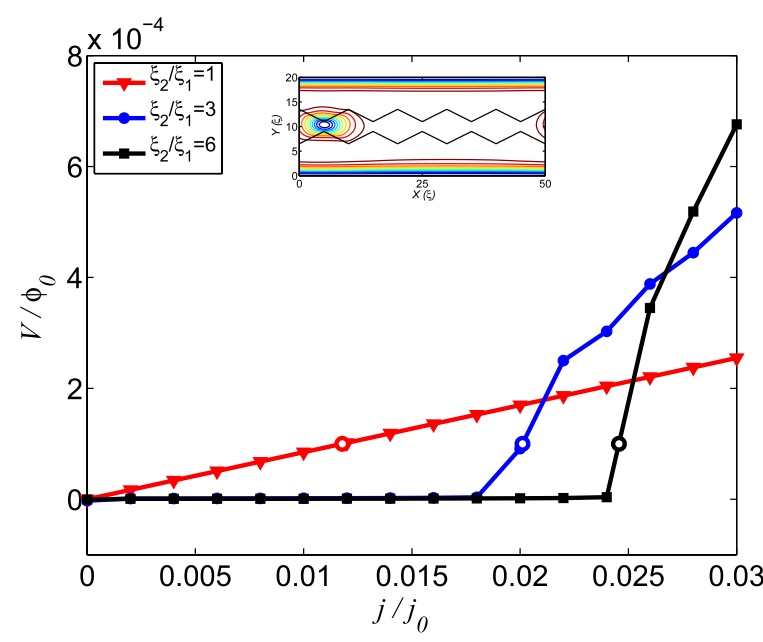

Figure 8. The $I V$-curves of the system $(w=4 \xi, \operatorname{tg}(\alpha)=0.5)$ for different $\xi_{2} / \xi_{1}$ ratio (in an infinite sample): the inset shows the order parameter distribution in the sample with channel for $w=4 \xi, \xi_{2} / \xi_{1}=6$, at $j=0.032 j_{0}$, and magnetic field $H=0.025 H_{\mathrm{c} 2}$.

pinned vortices. However, this value is much less than that for the channel vortices. Indeed, figure 7(b) shows a smooth change of the $I V$-curve, with practically no flux-pinned to flux-flow threshold. Although still we can distinguish the 'critical currents' for various $w$, by defining a threshold voltage, e.g., $V_{0}=10^{-4} \phi_{0}$. Thus in figure 7(b), the defined critical currents are $j_{\mathrm{c}}=0.027 j_{0}$ for $w=0, j_{\mathrm{c}}=0.041 j_{0}$ for $w=4 \xi$, and $j_{\mathrm{c}}=0.047 j_{0}$ for $w=8 \xi$. Therefore, the effect of 'soft pinning' in this case still remains, although it is much less pronounced than in the above case when all the vortices are confined by the channel. However, even in the presence of interstitial vortices, changing the geometry of the diamond channel influences the vortex flow (see figure 7(b)).

The dynamics of the system strongly depends also on the material parameters, i.e., it is determined by the ratio of the coherence lengths in the channel and the bank, $\xi_{2} / \xi_{1}$. This dependence is illustrated by the $I V$-curves shown in figure 8 (here, we consider the situation when vortices are present only in the channel; adding interstitial vortices would smoothen the $I V$-curves similarly to the case shown in figure $7(\mathrm{~b})$ ). The $I V$-curves are presented in figure 8 for $\xi_{2} / \xi_{1}=3$ and $\xi_{2} / \xi_{1}=6$. As a reference, we also show the $I V$-curve for $\xi_{2} / \xi_{1}=1$, i.e., for a sample without channel. Clearly, in this case vortices are not pinned, and they can freely move when driven by any value of the transport current. Therefore, the curve for $\xi_{2} / \xi_{1}=1$ does not contain a part corresponding to flux-pinned regime.

For $\xi_{2} / \xi_{1}$ values other than one, the motion of vortices occurs in the channel. Interestingly, this motion is influenced by two factors. On the one hand, due to the difference in the material parameters, the vortex mobility in the channel is higher than outside the channel which favors larger velocity and thus voltage as compared to the case without channel. This trend can be seen in the flux-flow regime, i.e., for $j / j_{0}>0.025$ (see figure 8). However, on the other hand, the geometric constriction (i.e., the neck of the diamond channel) 
creates a pinning potential that traps a vortex resulting in non-zero critical current. Strikingly, a channel characterized by a larger value of $\xi_{2} / \xi_{1}$ (i.e., $\xi_{2} / \xi_{1}=6$ ) demonstrates a larger critical current than a channel with a lower $\xi_{2} / \xi_{1}$, in spite of the fact that vortex mobility in this channel is larger (which can be seen in the flux-flow regime for $j / j_{0}>0.027$ ). This is explained by a stronger pinning effect for higher 'contrast' between the material parameters in the channel and in the bank (i.e., the ratio $\xi_{2} / \xi_{1}$ ). This indicates that the geometric constriction appears to be more important for controlling the transition from flux-pinned to flux-flow regime than the material parameters while the latter factor determines the motion for higher values of the current above the critical value.

\subsection{Effect of commensurability}

We have demonstrated that the material parameters (i.e., the ratio $\xi_{2} / \xi_{1}$ ) and the geometry of the channel (i.e., the minimum channel width, $w$ ) have a pronounced effect on the flux dynamics in a superconductor with a diamond-like channel. In particular, we have shown that, by changing the ratio $\xi_{2} / \xi_{1}$ and the gap $w$, one can control the transition from flux-pinned to flux-flow regimes and the slope of the $I V$-curves (see figures 7 and 8). Further, we analyze the critical current in the system as a function of the external applied magnetic field, of $j_{\mathrm{c}}(H)$. The results of simulations of $j_{\mathrm{c}}(H)$ for three values of the channel width, $w=0,4 \xi$, and $8 \xi$ are shown in figure 9. Figure 9(a) represents the case when vortices are confined in the diamond channel only. The $j_{\mathrm{c}}(H)$-curves have a very peculiar view, namely, they consist of a set of plateaus. Each plateau corresponds to a fixed number of vortices in the channel, $N_{\mathrm{v}}^{\text {ch }}$. For example, for $w=$ 0 , the critical current, $j_{\mathrm{c}} / j_{0}$ has a constant large value $\left(j_{\mathrm{c}} / j_{0} \approx\right.$ 0.28 ) for a range of fields $H / H_{\mathrm{c} 2} \approx 0-0.028$. For this range of fields, no vortex is generated in the system, and the high value of $j_{\mathrm{c}}$ corresponds to the depairing current (recall that the critical current is defined as a current resulting in a jump of voltage above some threshold value, e.g., $V / \phi_{0}=10^{-4}$ ). It is worth noting that the value of $j_{\mathrm{c}}$ slightly decreases when the width of the channel increases. This is explained as follows. The depairing critical current $j_{\mathrm{c}}$ is inversely proportional to the coherence length $\xi: j_{\mathrm{c}} \propto 1 / \xi$. Therefore, the critical depairing current is lower in the channel than in the bank. Since a vortex extends also to the region of the bank, its critical depairing current is influenced by both the channel superconductor (characterized by $\xi_{2}=6$ ) and by the bank material (with $\xi_{1}=$ 1). Therefore, increasing the width of the channel reduces the effect of the bank superconductor characterized by a higher depairing current. As a result, the critical current decreases, as can be seen in figure 9.

For larger fields, $0.028 \lesssim H / H_{\mathrm{c} 2} \lesssim 0.04$, the value of the critical current drops by almost an order of magnitude. The analysis of the dynamical patterns shows that for this range of fields the system encounters one vortex per simulation cell, and the critical current corresponds to the depinning transition. Further increasing applied magnetic field up to $H / H_{\mathrm{c} 2} \approx 0.06$ results in a sequence of plateaus
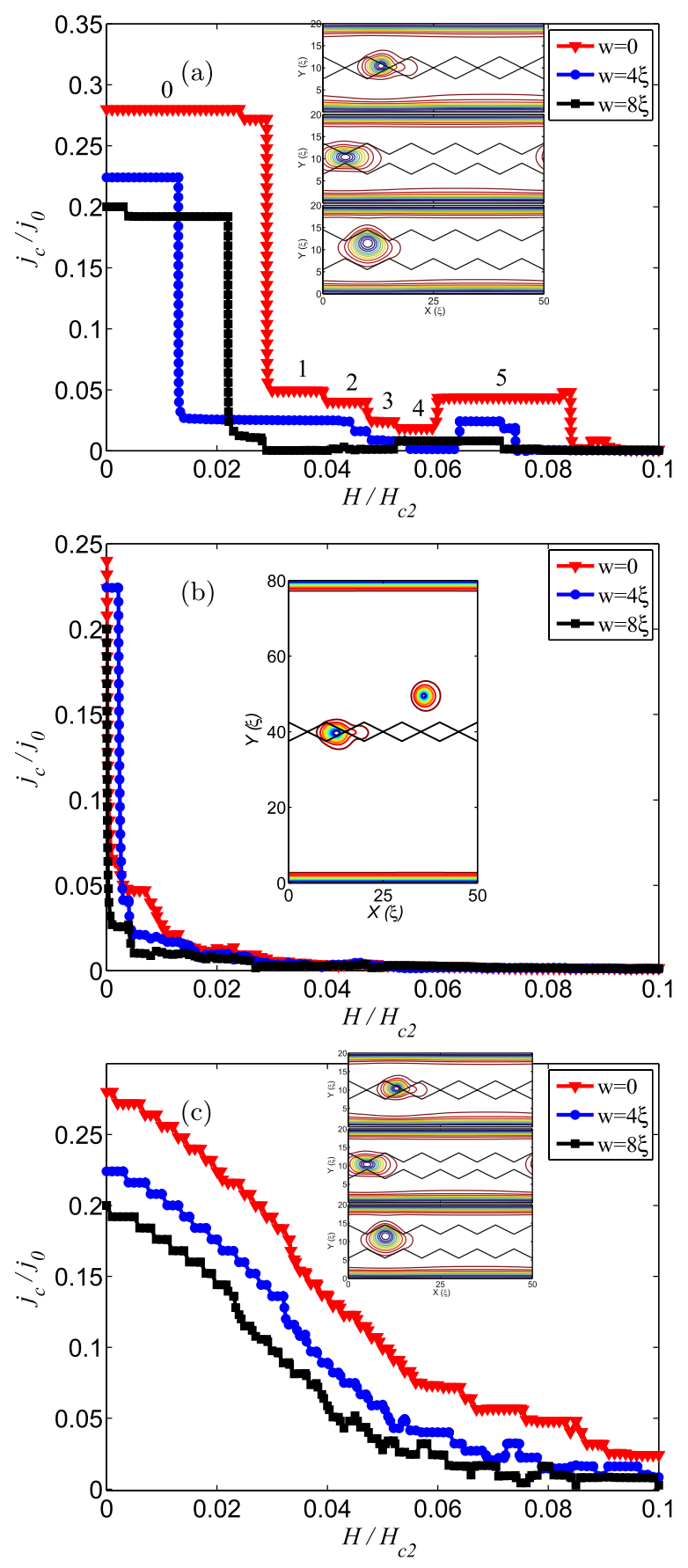

Figure 9. The critical current as a function of the magnetic field, $j_{\mathrm{c}}(H)$, for samples with $\xi_{2} / \xi_{1}=6$ and $\operatorname{tg}(\alpha)=0.5$, for varying channel gap. The system dimensions are: (a) $50 \xi \times 20 \xi$ (an infinite sample). Numbers near the plateaus in $j_{\mathrm{c}}$ show the corresponding number of vortices in the diamond channel; (b) $50 \xi \times 80 \xi$ (an infinite sample). Interstitial vortices lead to a strong suppression of the critical current and disappearance of the plateaus; (c) $50 \xi \times 20 \xi$ (a finite-size sample). The function $j_{\mathrm{c}}(H)$ shows a typical behavior for finite samples, i.e., decreasing with field.

of decreasing values in the function $j_{\mathrm{c}}(H)$ corresponding to $N_{\mathrm{v}}^{\text {ch }}=1-4$. However, this tendency is violated for $N_{\mathrm{v}}^{\text {ch }}=5$, i.e., when the vortex number matches to the cell number in the diamond channel. The critical current increases, which is a direct consequence of a commensurability effect. 


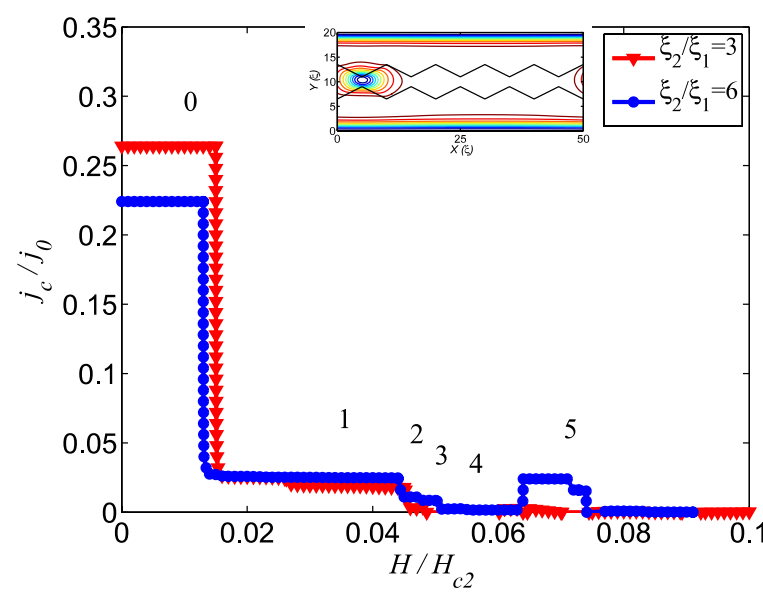

Figure 10. The critical current $j_{\mathrm{c}}(H)$ of the system with the cell dimensions $50 \xi \times 20 \xi$ (an infinite sample, $w=4 \xi, \operatorname{tg}(\alpha)=0.5$ ) for different ratios of the coherence lengths, $\xi_{2} / \xi_{1}$. Numbers near $j_{\mathrm{c}}$-plateaus show the number of vortices in the diamond channel.

When the number of vortices in the channel becomes larger than the cell number, the critical current rapidly decreases. A similar behavior is observed for wider channels, e.g., $w=4 \xi$ and $8 \xi$ (see figure 9(a)), however, the overall value of the critical current is considerably lower, and the commensurability peak is much less pronounced. Interstitial vortices easily destroy the observed structure of the $j_{\mathrm{c}}(H)$-curves: the critical current becomes much lower, and no commensurability features are revealed (see figure 9(b)). Note that the revealed commensurability peak (plateau) is also sensitive to the material parameters of the system. Thus, the commensurability peak found for $\xi_{2} / \xi_{1}=6$ disappears for a smaller value of the ratio, $\xi_{2} / \xi_{1}=3$ (see figure 10 ).

The demonstrated commensurability effect in diamond channels was also observed in the experiment [37]. Similarly, the critical current showed peaks for commensurate numbers of vortices in channels and channel cells. Note that in the experiments the $j_{\mathrm{c}}(H)$-curves were smooth which can be attributed to: (i) large channels containing tens of diamond cells, and (ii) presence of multiple channels in the sample (which leads to inhomogeneous broadening, due to a dispersion of the number of vortices in different channels). The effect of smoothing of the $j_{\mathrm{c}}(H)$-curves is also observed in finite samples (note that the $j_{\mathrm{c}}(H)$-curves shown in figures 9(a) and (b) were calculated for infinite channels, i.e., employing periodic boundary conditions). However, in this case the shape of $j_{\mathrm{c}}(H)$-curves is dominated by the barriers at the boundaries of the channel leading to the typical decreasing $j_{\mathrm{c}}(H)$ behavior [36].

The critical current can be controlled not only by the width of the channel, i.e., the gap, $w$, and the ratio of the coherence lengths inside and outside the channel, $\xi_{2} / \xi_{1}$, but also by the shape of the diamond cells. To analyze the dependence of the critical current $j_{\mathrm{c}}$ on the shape of the channel compartments, we calculated the critical current for channels with $\operatorname{tg} \alpha=0,1$, and 1.5 (angle $\alpha$ is defined in figure 1 , and channels with varying $\alpha$ are sketched in figure 11(a)). The corresponding function $j_{\mathrm{c}}(H)$ is shown in

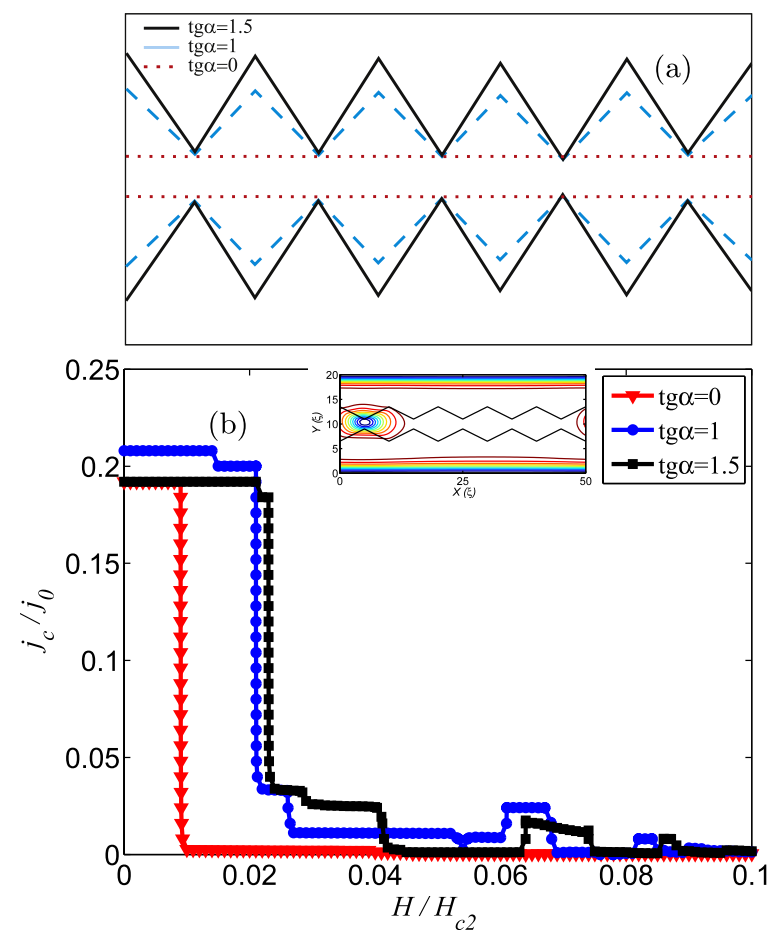

Figure 11. Schematic view of the channel with different $\alpha$. The critical current $j_{\mathrm{c}}(H)$ of the system with the cell dimensions $50 \xi \times 20 \xi$ (an infinite sample, $w=4 \xi, \xi_{2} / \xi_{1}=6$ ) for channel shapes characterized by different $\operatorname{tg}(\alpha)$.

figure 11(b). One can expect that increasing $\alpha$ should lead to a higher mobility of vortices in the channel, since larger $\alpha$ means increase in the effective width of the channel. On the other hand, increasing $\alpha$ means increasing steepness of the diamond cells, which prevents the motion of the vortices inside the channel. As a result, the dependence of $j_{\mathrm{c}}$ on $\operatorname{tg} \alpha$ can be non-monotonic which is observed for magnetic fields in the range $0.03<H / H_{\mathrm{c} 2}<0.04$ and for the commensurability peak values, $H / H_{\mathrm{c} 2} \approx 0.065$ (see figure $11(\mathrm{~b})$ ).

Thus we have demonstrated that the critical current $j_{\mathrm{c}}$ in a diamond channel can be controlled by the material parameter of the channel as well as by its geometry including the width of the channel and the shape of the diamond compartments.

\subsection{Negative $w$}

Finally, let us consider the case when the channel gap $w$ becomes negative. In this case, a continuous channel appears to be broken and turns to a chain of individual rhombus-shaped pinning sites.

In figure 12(a), we show a set of $I V$-curves for positive and negative $w$, changing from $w=8 \xi$ to $-4 \xi$. We found that changing $w$ to small negative values $(w=-2 \xi)$, first results in a slight increase of the critical depinning current since the neck between adjacent compartments becomes thicker. However, for larger negative $w(w=-4 \xi)$, the critical current decreases.

This behavior is explained by the fact that vortices are not point-like objects. The screening currents of the vortices extend over distances of the order of $\lambda$ which are greater than 

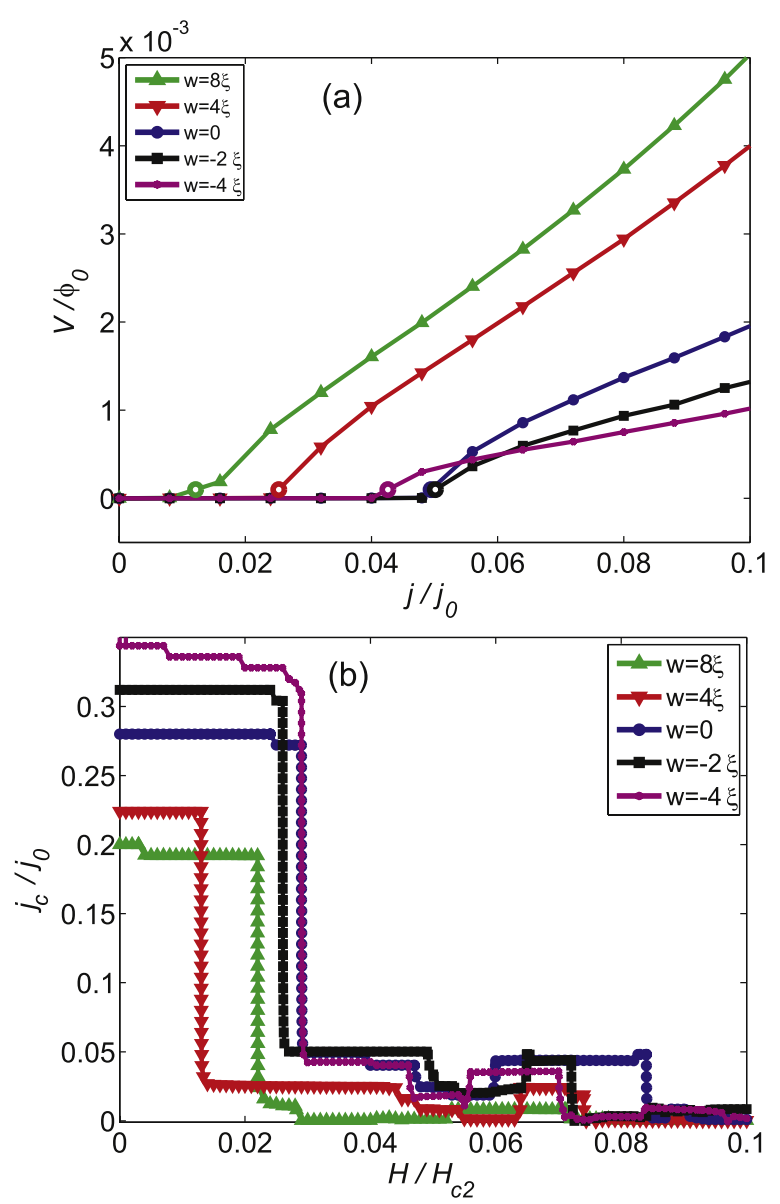

Figure 12. (a) Comparison of the $I V$-curves of the system for positive and negative values of the gap $w$. (b) The critical current as a function of the magnetic field, $j_{\mathrm{c}}(H)$, for positive and negative values of the gap $w$.

the compartment size. Therefore, the vortices are 'pinned' due to deformations of those screening currents (as discussed above), and the size of the neck appears to be less important than the size of the compartment (which results just in a slight increase of $j_{\mathrm{c}}$ ). For larger negative $w$, the compartments themselves become smaller. Thus a smaller fraction of the screening current appears to be inside the compartment, and, therefore, the 'pinning' efficiency decreases. This leads to the observed decrease in $j_{\mathrm{c}}$. The corresponding critical currents $j_{\mathrm{c}}$ as a function of the applied magnetic field $H$ are shown in figure 12(b).

\section{Conclusions}

By means of the time-dependent Ginzburg-Landau equations, we have investigated the dynamics of vortices driven in a 'diamond channel', i.e., a channel formed by superconducting material characterized by the critical temperature $T_{2}$ and the coherence length $\xi_{2}$ in shape of diamond-like cells inserted in another superconductor (bank) characterized by $T_{1}$ and $\xi_{1}$. We analyzed the conditions when generated vortices occupy only the channel and then they can also be generated in the banks. Depending on this, the dynamics of driven vortices is very different: the presence of interstitial vortices results in the disappearance of flux-pinned regime and smoothing the $I V$-characteristics of the device. The most interesting is thus the situation when vortices are present only in the channel. In this case, the diamond channel acts as a 'soft-pinning' device. Unlike in a linear array of individual pinning centers, the pinning potentials created by diamond-shaped compartments overlap and create a periodically modulated pinning channel. Thus this device is able to guide the flux and at the same time control the dynamical properties such as the transition from flux-pinned to flux-flow regime. The latter, as we show, can be reached by changing either the material parameters of the device (i.e., the ratio of the coherence lengths inside and outside the channel, $\xi_{2} / \xi_{1}$ ) or by the geometry of the channel (i.e., the degree of overlapping of the compartments defining the minimum channel width, $w$, and the specific shape of the diamond cells). We found that the critical current $j_{\mathrm{c}}$ of this device manifests striking commensurability features which were also observed in recent experiments [37].

Therefore, we have demonstrated that a 'diamond channel' can be a promising candidate for potential use in devices controlling magnetic flux motion.

\section{Acknowledgment}

This work was supported by the 'Odysseus' Program of the Flemish Government and the Flemish Science Foundation (FWO-V1).

\section{References}

[1] Bean C P 1962 Phys. Rev. Lett. 8250

[2] Richardson R A, Pla O and Nori F 1994 Phys. Rev. Lett. 721268

[3] Baert M, Metlushko V V, Jonckheere R, Moshchalkov V V and Bruynseraede Y 1995 Phys. Rev. Lett. 743269

[4] Moshchalkov V V, Baert M, Metlushko V V, Rosseel E, Van Bael M J, Temst K, Jonckheere R and Bruynseraede Y 1996 Phys. Rev. B 547385

[5] Bezryadin A, Ovchinnikov Y N and Pannetier B 1996 Phys. Rev. B 538553

[6] Castellanos A M, Wördenweber R, Ockenfuss G, Hart A v d and Keck K 1997 Appl. Phys. Lett. 71962

[7] Wördenweber R, Dymashevski P and Misko V R 2004 Phys. Rev. B 69184504

[8] Zhu B Y, Marchesoni F, Moshchalkov V V and Nori F 2003 Phys. Rev. B 6814514

Zhu B Y, Marchesoni F, Moshchalkov V V and Nori F 2003 Physica C 388/389 665

Zhu B Y, Marchesoni F, Moshchalkov V V and Nori F 2004 Physica C 404260

[9] Zhu B Y, Van Look L, Moshchalkov V V, Marchesoni F and Nori F 2003 Physica E 18322

[10] Zhu B Y, Marchesoni F and Nori F 2003 Physica E 18318

Zhu B Y, Marchesoni F and Nori F 2004 Phys. Rev. Lett. 92180602

[11] Marchesoni F, Zhu B Y and Nori F 2003 Physica A 32578

[12] Savel'ev S, Marchesoni F and Nori F 2003 Phys. Rev. Lett. 9110601

Savel'ev S, Marchesoni F and Nori F 2004 Phys. Rev. Lett. 92160602

[13] Nori F 2006 Nature Phys. 227 
[14] Misko V R, Savel'ev S, Rakhmanov A L and Nori F 2006 Phys. Rev. Lett. 96127004

Misko V R, Savel'ev S, Rakhmanov A L and Nori F 2007 Phys. Rev. B 75024509

[15] Villegas J E, Savel'ev S, Nori F, Gonzalez E M, Anguita J V, García R and Vicent J L 2003 Science 3021188

[16] Van Look L, Zhu B Y, Jonckheere R, Zhao B R, Zhao Z X and Moshchalkov V V 2002 Phys. Rev. B 66214511

[17] Silhanek A V, Raedts S, Lange M and Moshchalkov V V 2003 Phys. Rev. B 67064502

[18] Selders P and Wördenweber R 2000 Appl. Phys. Lett. 763277

[19] Misko V, Savel'ev S and Nori F 2005 Phys. Rev. Lett. 95177007

[20] Misko V R, Savel'ev S and Nori F 2006 Phys. Rev. B 74024522

[21] Kramer R B G, Silhanek A V, Van de Vondel J, Raes B and Moshchalkov V V 2009 Phys. Rev. Lett. 103067007

[22] Kemmler M, Gürlich C, Sterck A, Pöhler H, Neuhaus M, Siegel M, Kleiner R and Koelle D 2006 Phys. Rev. Lett. 97147003

[23] Misko V R, Bothner D, Kemmler M, Kleiner R, Koelle D, Peeters F M and Nori F 2010 Phys. Rev. B 28184512

[24] Misko V R and Nori F 2012 Phys. Rev. B 85184506

[25] Motta M, Colauto F, Ortiz W A, Fritzsche J, Cuppens J, Gillijns W, Moshchalkov V V, Johansen T H, Sanchez A and Silhanek A V 2013 Appl. Phys. Lett. 102212601

[26] Silhanek A V, Gillijns W, Moshchalkov V V, Zhu B Y, Moonens J and Leunissen L H A 2006 Appl. Phys. Lett. 89152507

[27] Wambaugh J F, Reichhardt C, Olson C J, Marchesoni F and Nori F 1999 Phys. Rev. Lett. 835106

[28] Lin N S, Heitmann T W, Yu K, Plourde B L T and Misko V R 2011 Phys. Rev. B 84144511

[29] Van de Vondel J, de Souza Silva C, Zhu B Y, Morelle M and Moshchalkov V V 2005 Phys. Rev. Lett. 94057003

[30] Sboychakov A O, Savel'ev S, Rakhmanov A L and Nori F 2010 Phys. Rev. Lett. 104190602

[31] Derényi I 2002 Appl. Phys. A 57217

[32] Togawa Y, Harada K, Akashi T, Kasai H, Matsuda T, Nori F, Maeda A and Tonomura A 2005 Phys. Rev. Lett. 95087002

[33] Savel'ev S, Misko V R, Marchesoni F and Nori F 2005 Phys. Rev. B 71214303

[34] Silhanek A V, Van de Vondel J, Moshchalkov V V, Leo A, Metlushko V, Ilic B, Misko V R and Peeters F M 2008 Appl. Phys. Lett. 92176101
[35] Kokubo N, Sorop T G, Besseling R and Kes P H 2006 Phys. Rev. B 73224514

[36] Plourde B L T, Van Harlingen D J, Vodolazov D Y, Besseling R, Hesselberth M B S and Kes P H 2001 Phys. Rev. B 64014503

[37] Yu K, Hesselberth M B S, Kes P H and Plourde B L T 2010 Phys. Rev. B 81184503

[38] Reichhardt C and Nori F 1999 Phys. Rev. Lett. 82414

[39] Silhanek A V, Van Look L, Raedts S, Jonckheere R and Moshchalkov V V 2003 Phys. Rev. B 68214504

[40] Vestgården J I, Yurchenko V V, Wördenweber R and Johansen T H 2012 Phys. Rev. B 85014516

[41] Wambaugh J W, Marchesoni F and Nori F 2003 Phys. Rev. B 67144515

[42] Lima C L S and de Souza Silva C 2009 Phys. Rev. B 80054514

[43] Kapra A V, Misko V R, Vodolazov D Y and Peeters F M 2011 Supercond. Sci. Technol. 24024014

[44] Weber A and Kramer L 1991 J. Low Temp. Phys. 84289

[45] Kramer L and Watts-Tobin R J 1978 Phys. Rev. Lett. 401041

[46] Ivlev B I and Kopnin N B 1984 Sov. Phys.-Usp. 27206

[47] Gladilin V N, Tempere J, Devreese J T, Gillijns W and Moshchalkov V V 2009 Phys. Rev. B 80054503

[48] Gropp W D, Kaper H G, Leaf G K, Levine D M, Palumbo M and Vinokur V M 1996 J. Comput. Phys. 123254

[49] Vodolazov D Y, Peeters F M, Grigorieva I V and Geim A K 2005 Phys. Rev. B 72024537

[50] Vodolazov D Y, Baelus B J and Peeters F M 2004 Physica C 404400

[51] Meissner W and Ochsenfeld R 1933 Naturwissenschaften 21787

[52] Schweigert V A, Peeters F M and Deo P S 1998 Phys. Rev. Lett. 812783

[53] Geim A K, Dubonos S V, Grigorieva I V, Novoselov K S, Peeters F M and Schweigert V A 2000 Nature 40755

[54] Grigorieva I V, Escoffier W, Richardson J, Vinnikov L Y, Dubonos S and Oboznov V 2006 Phys. Rev. Lett. 96077005

[55] Grigorieva I V, Escoffier W, Misko V R, Baelus B J, Peeters F M, Vinnikov L Y and Dubonos S 2007 Phys. Rev. Lett. 99147003

[56] Chibotaru L F, Ceulemans A, Bruyndoncx V and Moshchalkov V V 2000 Nature 408833

[57] Misko V R, Fomin V F, Devreese J T and Moshchalkov V V 2003 Phys. Rev. Lett. 90147003

[58] Dikin D A, Chandrasekhar V, Misko V R, Fomin V M and Devreese J T 2003 Eur. Phys. J. B 34231 